\title{
BCAR1 Protein Plays Important Roles in Carcinogenesis and Predicts Poor Prognosis in Non-Small-Cell Lung Cancer
}

\author{
Wei Huang, Bo Deng*, Ru-Wen Wang*, Qun-You Tan, Yong He, Yao-Guang Jiang, Jing-Hai Zhou
}

Thoracic Surgery Department, Institute of Surgery Research, Daping Hospital, Third Military Medical University, Chongqing, People's Republic of China

\begin{abstract}
Objective: Our previous study suggested the potential clinical implications of BCAR1 in non-small-cell lung cancer (NSCLC) (Mol Diagn Ther. 2011. 15(1): 31-40). Herein, we aim to evaluate the predictive power of BCAR1 as a marker for poor prognosis in NSCLC cases, verify the carcinogenic roles of BCAR1 in the A549 lung adenocarcinoma cell line, and testify to the BCAR1/phospho-p38 axis.

Methods: Between January 2006 and June 2010, there were a total of 182 patients with NSCLC (151 cases with available follow up data, and 31 cases lost to follow-up due to the invalid contact information). We inspected BCAR1, phosphoBCAR1(Tyr410), phospho-p38(Thr180/Tyr182) and p38 expression in NSCLC tissues and matched adjacent normal tissues by immunoblotting and IHC. After BCAR1 -RNA interference in A549 cells, we inspected the protein expression (BCAR1, phospho-BCAR1, phospho-p38 and p38) and performed cell biology experiments (cell growth, migration and cycle).
\end{abstract}

Results: BCAR1 was overexpressed in NSCLC tissues (177/182) and cell lines (A549 and Calu-3). However, it was not detected in the normal adjacent tissue in 161 of the 182 cases. Higher BCAR1 levels were strongly associated with more poorly differentiated NSCLC and predicted poorer prognosis. BCAR1 knockdown caused cell growth arrest, cell migration inhibition and cell cycle arrest of A549 cells. Overexpression of BCAR1 was associated with activation of p38 in NSCLC cases, and BCAR1 knockdown caused reduction of phospho-p38 levels in A549 cells.

Conclusion: Overexpression of BCAR1 is a predictor of poor prognosis in NSCLC and plays important carcinogenic roles in carcinogenesis, probably via activation of p38 MAPK. However, further investigations are required immediately.

Citation: Huang W, Deng B, Wang R-W, Tan Q-Y, He Y, et al. (2012) BCAR1 Protein Plays Important Roles in Carcinogenesis and Predicts Poor Prognosis in NonSmall-Cell Lung Cancer. PLoS ONE 7(4): e36124. doi:10.1371/journal.pone.0036124

Editor: Keiran Smalley, The Moffitt Cancer Center \& Research Institute, United States of America

Received December 16, 2011; Accepted March 26, 2012; Published April 27, 2012

Copyright: (C) 2012 Huang et al. This is an open-access article distributed under the terms of the Creative Commons Attribution License, which permits unrestricted use, distribution, and reproduction in any medium, provided the original author and source are credited.

Funding: The work was supported by the National Natural Science Foundation of China (NSFC) (No. 81101782), NSFC project CQ CSTC (No. CSTC2011BB5020), and NSFC Third Military Medical University (No. 2010XQN36). The funders had no role in study design, data collection and analysis, decision to publish, or preparation of the manuscript.

Competing Interests: The authors have declared that no competing interests exist.

*E-mail: superdb@163.com (BD); wangrw53@126.com (RW)

\section{Introduction}

Every year, there are 1.35 million new lung cancer cases in the world [1]. Worldwide, Lung cancer is the leading cause of cancerrelated deaths [2]. Due to the intricate biological functions, prognosis of lung cancer remains very poor [1]. Hence, it is vital to unveil the biological functions of the disease for the sake of improving therapeutic efficacy [3].

Breast cancer anti-estrogen resistance 1 (BCAR1), also entitled p130cas, was one of the CAS protein (Crk-associated substrate) family members. It was originally identified as a cellular protein migrating at $130 \mathrm{kDa}$, and to be hyperphosphorylated in v-Crk and $\mathrm{v}$-Src transformed cells [4,5]. Initially, intensive studies were focused on the correlation between breast cancer and BCAR 1 $[6,1,7,8]$. For instance, Brinkman et al. [7] suggested BCAR1 overexpression in ZR-75-1 breast cancer cell line renders antiestrogen resistance to the cells. Furthermore, Dorssers et al. [8] reported that BCARl expression was inversely related to relapse-free survival and overall survival time of breast cancer.
Recently, our study suggested there is a clinical implication of BCAR1 in non-small-cell lung cancer (NSCLC) [9]. We investigated serum BCARl levels in 80 NSCLC cases and 80 healthy controls, respectively, by using a specific enzyme-linked immunosorbent assay (ELISA) [9]. Intriguingly, we found that serum BCAR1 levels were significantly higher in NSCLC than in the control group, increased gradually with the progression of tumor staging, and decreased after removal of the malignant lesions [9]. However, the oncogenic mechanisms of BCARl in NSCLC are still the enigmas.

Herein, we conducted the further investigations to evaluate the predictive power of BCARl as a biomarker for poor prognosis in NSCLC patients. And we verified the carcinogenic roles of BCAR1 via RNA interference (RNAi) in A549 lung adenocarcinoma cell line. Experiments in vivo and vitro demonstrated the closed correlation between BCARl expression and activation of p38 which is a crucial branch of the MAPK (mitogen-activated protein kinase) pathway $[10,11]$. 


\section{Materials and Methods}

\section{Patients}

The study protocol was reviewed and approved by the Research Ethics Board in Daping hospital (Chongqing City, P.R.China) [reference no. TMMU-DPH/2006-012], and informed consent was written and obtained from all the patients.

In the study performed between January 2006 and June 2010, there were a total of 182 patients with NSCLC. Pulmonary neoplasm was diagnosed radiographically and confirmed by pathology. None of the patients had received treatment before enrollment in the study. The demographic and clinicopathological characteristics of patients are shown in table 1. All the patients underwent lobectomy and lymphadenectomy. One hundred and thirty five patients received postoperative adjuvant chemotherapy (Paclitaxel plus Nedaplatin). Postoperative follow-up was available in 151 cases by telephone or letter interview, and 31 cases lost to follow-up due to the invalid contact information.

\section{Cell Culture}

A549 and Calu-3 lung adenocarcinoma cell lines were obtained from the American Type Culture Collection and cultured in RPMI1640/10\% FBS, respectively.

\section{RNA Interference of BCAR1}

Firstly, the following oligoribonucleotide pairs were used: 5 'CGGGGGTCGACAGTGGTGTGTATTTCAAGAGAATACACACGACTGTCGACGTTTTTTg-3' and 5'-AATTCAAAAAAGGTGGACAGTGGTGTGTATTCTCTTGAAATAGACACCACTGTCGACG-3'. Entire sequences were derived from the sequence of human BCAR1 mRNA. The oligonucleotides were obtained from Sunbio Medical Biotechnology CO., Ltd (Shanghai City, P.R.China). The complementary two strands (each at $20 \mu \mathrm{M}$ ) in $60 \mu \mathrm{l}$ of annealing buffer (Sunbio Medical Biotechnology CO., Ltd, Shanghai City, P.R.China) were heated for $5 \mathrm{~min}$ at $95^{\circ} \mathrm{C}$ and then incubated for $1 \mathrm{~h}$ at room temperature. Thereafter, the GFP-tagged lentiviral vector pLVT351.LV for BCARl-RNAi was constructed by inserting the annealing

Table 1. The patients' clinical and pathological characteristics according to BCAR1 and p-p38 expression in NSCLC.

\begin{tabular}{|c|c|c|c|c|c|c|c|c|c|c|c|}
\hline \multirow{2}{*}{ Characteristics } & \multirow{2}{*}{ No. } & \multicolumn{5}{|c|}{ BCAR1 } & \multicolumn{5}{|c|}{ p-p38 } \\
\hline & & - & + & + & +1 & $P$ & - & + & $H$ & ++ & $P$ \\
\hline \multicolumn{12}{|l|}{ Group } \\
\hline NSCLC & 182 & 5 & 75 & 60 & 42 & $0.000 \#$ & 101 & 36 & 32 & 13 & $0.000 \#$ \\
\hline Normal tissue & 182 & 156 & 26 & 0 & 0 & & 148 & 32 & 2 & 0 & \\
\hline \multicolumn{12}{|l|}{ Age } \\
\hline$<60$ & 78 & 3 & 31 & 30 & 14 & $0.507^{*}$ & 49 & 12 & 12 & 5 & $0.129^{*}$ \\
\hline$\geq 60$ & 104 & 2 & 44 & 30 & 28 & & 52 & 24 & 20 & 8 & \\
\hline \multicolumn{12}{|l|}{ Gender } \\
\hline Female & 56 & 1 & 16 & 22 & 17 & $0.16^{*}$ & 31 & 11 & 7 & 7 & $0.792^{*}$ \\
\hline Male & 126 & 4 & 59 & 38 & 25 & & 70 & 25 & 25 & 6 & \\
\hline \multicolumn{12}{|l|}{ Histology } \\
\hline squamous cell carcinoma & 115 & 4 & 51 & 37 & 23 & $0.11^{*}$ & 68 & 20 & 20 & 7 & $0.242^{*}$ \\
\hline adenocarcinomas. & 67 & 1 & 24 & 23 & 19 & & 33 & 16 & 12 & 6 & \\
\hline \multicolumn{12}{|l|}{ Differentiation } \\
\hline Well & 63 & 0 & 37 & 18 & 8 & $0.010 \triangle$ & 37 & 11 & 11 & 4 & $0.665 \triangle$ \\
\hline Moderately & 97 & 4 & 32 & 34 & 27 & & 53 & 20 & 17 & 7 & \\
\hline Poorly & 22 & 1 & 6 & 8 & 7 & & 11 & 5 & 4 & 2 & \\
\hline \multicolumn{12}{|l|}{ TNM stage } \\
\hline I & 41 & 0 & 21 & 16 & 4 & $0.241 \triangle$ & 23 & 7 & 8 & 3 & $0.889 \triangle$ \\
\hline II & 91 & 3 & 35 & 30 & 23 & & 48 & 22 & 14 & 7 & \\
\hline III & 47 & 2 & 17 & 13 & 15 & & 28 & 7 & 9 & 3 & \\
\hline IV & 3 & 0 & 2 & 1 & 0 & & 2 & 0 & 1 & 0 & \\
\hline \multicolumn{12}{|l|}{ lymphonode status } \\
\hline NO & 128 & 4 & 54 & 40 & 30 & $0.711^{*}$ & 71 & 24 & 23 & 10 & $0.818^{*}$ \\
\hline N1-N3 & 54 & 1 & 21 & 20 & 12 & & 30 & 12 & 9 & 3 & \\
\hline \multicolumn{12}{|l|}{ Tumor size } \\
\hline$<3 \mathrm{~cm}$ & 101 & 3 & 48 & 26 & 24 & $0.206^{*}$ & 57 & 21 & 16 & 7 & $0.523^{*}$ \\
\hline$\geq 3 \mathrm{~cm}$ & 81 & 2 & 27 & 34 & 18 & & 44 & 15 & 18 & 6 & \\
\hline
\end{tabular}

Note:

$\#: X^{2}$ test for the positive rate of staining (NSCLC vs Normal adjacent tissue);

*: Mann-whitney U test;

$\triangle$ : Kruskal-Wallis test.

doi:10.1371/journal.pone.0036124.t001 
nucleotides into the Age I+EcoR I site of pMAGic 4.1 (Sunbio Medical Biotechnology CO., Ltd, Shanghai City, P.R.China).

Secondly, A549 cell line was plated at $2.3 \times 10^{5}$ cells per well of 24-well cell culture plate and infected with lentivirus at a multiplicity of infection (MOI) of 10. Cells infected with pLVT351-L.V. and CMV-GFP-L.V.(blank lentiviral vector, pMAGic 4.1) was named as A549-BCAR1-RNAi and A549negative control, respectively.

\section{Western Blotting analysis of BCAR1, phospho-BCAR1, phospho-p38 and p38 in NSCLC tissues and cells}

NSCLC and matched adjacent normal tissues (at least $5 \mathrm{~cm}$ away from the primary tumors) were available from a total of sixty (including 35 adenocarcinomas and 25 squamous cell carcinomas) cases for western blotting. During the operations, the samples were collected by the technicians promptly following removals. Besides, we also prepared the cell lines including Calu-3, A549, A549negative control and A549-BCAR1-RNAi.

Thereafter, lysates from tissues and cell lines were prepared in a RIPA buffer comprising $50 \mathrm{mM}$ Tris- $\mathrm{HCl}$ pH 7.5, $150 \mathrm{mM}$ $\mathrm{NaCl}, 1 \%$ Triton X-100, 0.1\% SDS, 0.5\% deoxycholic acid and $0.02 \%$ sodium azide. The protein concentrations were determined with a BCA Protein Assay Kit (Pierce, Rockford, IL).

Proteins were denatured at $95^{\circ} \mathrm{C}$ for $5 \mathrm{~min}$, and $50 \mu \mathrm{g}$ protein per lane was resolved by sodium dodecyl sulfate-polyacrylamide gel electrophoresis (SDS-PAGE) using 10\% polyacrylamide gel. Proteins were blotted on polyvinylidene difluoride (PVDF) membranes (Thermo), which were then blocked with 5\% skim milk for $1 \mathrm{~h}$ at room temperature. The proteins were immunoblotted using anti-BCAR1 antibody(BD Transduction Laboratories, USA, 1:1000), anti-phospho-BCARl (Tyr410) antibody (Abcam, USA, 1:1000), anti-p38 (BD Transduction Laboratories material, USA, 1:1000) and anti-phospho-p38(Thr180/Tyr182) antibody (cell signaling Transduction Laboratories, USA, 1:1000). An anti- $\beta$-actin(Sigma) or GAPDH (Sigma) antibody served as the control.

Gray scales of immunoblotting of NSCLC and matched adjacent normal tissues were quantitatively analyzed by using image acquisition and analysis software (Image Lab software, BIORAD, USA) according to the manufacturer's instructions.

\section{Tissue microarray construction and Immunohistochemical (IHC) assay of BCAR1, phospho- BCAR1, p38 and phospho-p38 in NSCLC tissues}

The hematoxylin and eosin (H\&E)-stained slides of all 182 cases were inspected. For each case, the pathologic diagnosis was identified on the $\mathrm{H} \& \mathrm{E}$-stained slide and then circled. A corresponding slide of normal adjacent tissue was also marked. Tissue microarray was constructed according to the previously published protocols [9]. The marked slide was aligned with the surface of the corresponding donor block. Thereafter, the area was marked on the paraffin tissue blocks. From each specimen, tissue cores with a diameter of $1.5 \mathrm{~mm}$ were punched and then arrayed on a recipient paraffin block. Sections $(4 \mathrm{~mm})$ of these microarray blocks were cut and then used for the IHC analysis.

The sections were incubated with serum blocking solution and primary antibodies including anti-BCAR1 antibody (BD Transduction Laboratories, USA, 1:100), anti-phospho-BCAR1 (Tyr410) antibody (Abcam, USA, 1:100), anti-p38 antibody (BD Transduction Laboratories, USA, 1:50) and anti-phospho-p38 (Thr180/Tyr182) antibody (cell signaling Transduction Laboratories, USA, 1:10), biotinylated secondary antibody, and streptavidin-horseradish peroxidase. Diaminobenzidine solution was used as a chromogen. The slides were then counterstained in a hematoxylin solution. The IHC results on protein abundance were classified according to the percents of positive cells as follows: negative staining; + , weak position $(<25 \%)$; ++, moderate position $(<50 \%)$; +++, strong position ( $\geq 50 \%)$. " $<25 \%$ " was classified as low expression, otherwise as high expression. Three observers carried out to score the slides and mean values were obtained finally.

\section{TDT-mediated dUTP nick end labeling assay (TUNEL) of NSCLC tissues}

We conducted TUNEL assay in tissue sections of all the 182 cases. Apoptosis in the tumor was inspected with In Situ Cell Death Detection kit, POD (Roche, Germany). Tissue sections were deparaffinize in xylene, and hydrated through graded ethanol and pretreated with microwave antigen retrieval (citrate buffer, $\mathrm{pH}$ 7.5). Endogenous peroxidase was blocked by $0.3 \%$ $\mathrm{H}_{2} \mathrm{O}_{2}$ in methanol for 15 min. Thereafter, tissue sections were treated with $3 \%$ bovine serum albumin for $30 \mathrm{~min}$ and incubated with TUNEL reaction mixture (TDT : dUTP, 1:20) for $45 \mathrm{~min}$ at $37^{\circ} \mathrm{C}$. Tissue sections were combined with Converter-POD, followed by washing and DAB (Zhong Shong, China) color reaction. During the TUNEL procedure, sections were washed in phosphate buffer saline (PBS). Tissue sections were counterstained by hematoxylin, dehydrated through graded ethanol, cleared in xylene, and mounted. For negative controls, deionized water was used instead of TDT. Positive controls consisted of inflamed human tonsil. Cells were considered positive when intense brown reactivity was detected in the nuclei. Apoptotic index was calculated the percentage of nuclear positive stain in 1000 cells in five different sites of each section at a $400 \times$ magnification.

\section{Real-time RT-PCR}

To evaluate the efficacy of RNAi of BCAR1, we performed quantitative real-time RT-PGR in A549-BCAR1-RNAi and A549-negative control cells. Either of the cells was seeded at a concentration of $1 \times 10^{5}$ cells/well in 6 -well plates. After two days seeding, total RNA was extracted from cells using Trizol reagent (Invitrogen). First-strand cDNA was synthesized with M-MLV transcriptase (Promega) and oligo dT. Real-time PCR was performed using SYBR Green PCR master mix (TAKARA) and the ABI Prism 7000 sequence detection system (Applied Biosystems, Foster City, CA). PGR primers were used as followings: 5'-CAATGCGTCACTGCTCTT-3' and 5'-GTAGTCATAGTCGTCGATC-3'. The specificity of detected signals was confirmed by a dissociation curve consisting of a single peak. All samples were run in duplicate in each experiment. Values were normalized by human $\beta$-actin.

\section{Cell Growth Assay}

For evaluation of cell growth, A549-negative control and A549BCAR 1-RNAi cells was plated at $2.0 \times 10^{2}$ cells per well in six-well plates, respectively. And either of the cells was inspected and photographed following Giemsa staining, eleven days after plating.

\section{Cell Migration Assay}

For assessment of cell motility, chamber migration assays were conducted using a cell culture insert $(8-\mu \mathrm{m}$ pore size, 24 -well format; Cell Invasion Assay Kit Cat. CHEMICON, No. EGM550). A549-negative control and A549-BCAR1-RNAi cells were seeded in duplicate at a density of $3.0 \times 10^{5}$ cells/ chamber. After 48 hours, cells which had not moved to the lower wells were removed from the upper face of the filters using cotton 
swabs, and cells that had moved to the lower surface of the filter were stained by using a Cell Invasion Assay Kit Cat. (CHEMICON, No. ECM550). Cell migration was quantified by visual counting after being photographed. Experiments were performed in duplicate. Mean values for three random fields were obtained for each well.

\section{Cell Cycle Assays}

For flow cytometric determination of cell cycles, $2.0 \times 10^{6}$ of A549-negative control cells and A549-BCAR1-RNAi cells were fixed in $70 \%$ ethanol and stained with propidium iodide, respectively. The stained cells were analyzed on a FACScan flow cytometer for relative cell cycles. Experiments were performed in duplicate.

\section{Cell Apoptosis Assays}

We evaluated cell apoptosis using FACScan flow cytometer. $2.0 \times 10^{6}$ of A549-negative control cells $(48 \mathrm{~h}$ after transient transfection), A549-negative control cells (stable transfection), A549-BCAR1-RNAi cells (48 h after transient transfection) and A549-BCAR1-RNAi cells (stable transfection) were fixed in $70 \%$ ethanol and stained with propidium iodide, respectively. The stained cells were analyzed on a FACScan flow cytometer for relative cell apoptosis. Experiments were performed in duplicate.

\section{Data Analysis}

The statistical analysis was performed using the Student's t-test, Mann-whitney U test, Kruskal-Wallis test, $X^{2}$ test and Spearman's rho, respectively. Death from any cause was included in the calculation of postoperative survival. The disease specific survival was calculated by the Kaplan-Meier method and analyzed by the log-rank test. Prognostic factors were examined by univariate and multivariate analyses using a Cox proportional hazards model. All of the aforesaid calculations were performed using SPSS Version 11.0 software for Windows (SPSS, Inc., Chicago, USA). A value of $\mathrm{p}<0.05$ (two-sided) was considered statistically significant.

\section{Results}

\section{BCAR1 is overexpressed in NSCLC tissues and cell lines}

BCAR1 expression was detected (either in the nucleus, the cytoplasm, or both) in 57 of the 60 NSCLC cases by using Immunoblotting (Figure 1a), and 177 of the 182 NSCLC cases by using IHC assay(Figure 1c), respectively. However, it was not detected in the normal adjacent tissue in 53 of the 60 cases by using Immunoblotting (Figure 1a), and 161 of the 182 cases by using IHC assay (Figure not shown), respectively. Analysis of gray scales of immunoblotting also suggested BCARl levels were significantly higher in NSCLC than in the normal adjacent tissue (48.2 \pm 24.7 vs $11.0 \pm 9.8$, Student's t-test, $P<0.001$, Figure $1 \mathrm{~b})$.

However, phospho-BCAR 1(Tyr410) was detected in cytoplasm in 29 of the 60 NSCLC by using Immunoblotting (Figure 1a), and 32 cases of the 182 NSGLC cases by using IHC assay(Figure 1c), respectively. However, it was also detected in the normal adjacent tissue in 30 of the 60 cases by using Immunoblotting (Figure 1a), and 34 of the 182 cases by using IHC assay (Figure not shown), respectively. Analysis of gray scales of immunoblotting suggested there was no appreciable difference of BCAR1 levels in between NSGLC and the normal adjacent tissue (25.3 \pm 11.2 vs 27.8 \pm 15.2 , $P=0.476$, Student's t-test, Figure $1 \mathrm{~b})$.

BCAR1 and phospho-BCAR1(Tyr410) was detected in A549 and Calu-3 NSCLC cells by using immunoblotting assay (Figure 1a).
Higher BCAR1 levels are strongly correlated with more poorly differentiated tumors and predicts poorer

\section{prognosis}

By using IHC assay in the 182 NSCLC cases, we found that higher BCAR1 levels were strongly correlated with more poorly differentiation. (Kruskal-Wallis test, $P=0.01$; Table 1). However, there was no appreciable correlation between BCAR 1 and other clinical-pathological characteristics including age, gender, histology, TNM stage, tumor size and lymphonode metastasis (Table 1). Despite BCAR1 expression was detected either in cytoplasm, nucleus, or both in NSCLC, there was no appreciable correlation between the protein location and clinical-pathological parameters (Data not shown). Besides, there was no significant correlation between phospho-BCAR1 and the clinical-pathological characteristics (Data not shown).

The survival rate of BCARl high-expression group was significantly lower than of low-expression group $(P=0.001$, Figure 2a). Multivariate analysis revealed that BCARl levels (hazard ratio $1.777, P=0.028$ ), lymphonode metastasis (hazard ratio $1.277, P=0.040$ ) and TNM stage (hazard ratio 1.298, $P=0.007)$ were significant and independent prognostic indicators for NSCLC cases (Table 2).

\section{BCAR1 knockdown causes cell growth arrest, cell} migration inhibition and cell cycle arrest of A549 cells

We established human NSCLC cancer A549 cells that stably expressed pLVT351-L.V. (A549-BCAR1-RNAi cells) and CMVGFP-L.V. (A549-negative control cells) through use of a lentivirus system. At least an $80 \%$ reduction in mRNA levels of BCAR1 in A549-BCAR1-RNAi cells was confirmed by real-time RT-PCR (Figure 3a) and Western Blotting analysis (Figure 1a), respectively. Simultaneously, we can see the inhibition of phospho-BCAR 1 along with BCAR1 knockdown (Figure la).

Figure 3b suggested colony forming units of A549-BCAR1RNAi cells were detectably less than of A549-negative control cells, eleven days after plating (Figure 3b). Cell migration assay suggested BCAR1 knockdown prevented cell migration of A549BCAR1-RNAi cells (Figure 3c). And Flow cytometry indicated BCAR 1 knockdown also caused cell cycle arrest of A549-BCAR1RNAi cells (Figure 3d).

\section{BCAR1 is adversely correlated to apoptotic index in NSCLC tissues. However, BCAR1 knockdown can not increase apoptosis in A549 cells}

Apoptotic bodies were detected in all the 182 NSCLC tissues (Figure 1c). Among the tissues, there was a significant and inverse correlation between BCAR1 and apoptotic index (Spearman's rho, correlation coefficient $=-0.183 ; P=0.013)$. Apoptotic index in BCAR1 high-expressed tissues was substantially lower than in BCAR1 low-expressed tissues (Student's t-test $\mathrm{t}=2.312 P=0.022$ ).

However, in comparison with negative control cells, BCAR 1 knockout did not increase apoptosis rate following either transient $(39.1 \%$ vs $42.1 \%)$ or stable transfection $(0.33 \%$ vs $0.4 \%)$ in A549 cells (Figure 3e).

Overexpression of BCAR1 is correlated with activation of p38 in NSCLC cases and BCAR1 knockdown causes reduction of phospho-p38 in A549 cells

Phospho-p38 expression was detected in 31 of the 60 NSCLC cases by using immunoblotting (Figure 1a), and 91 of the 182 NSCLC cases (prone to locate in the nucleus) by using IHC assay (Figure 1c). Similar to the trend of BCAR1 levels, gray scales 


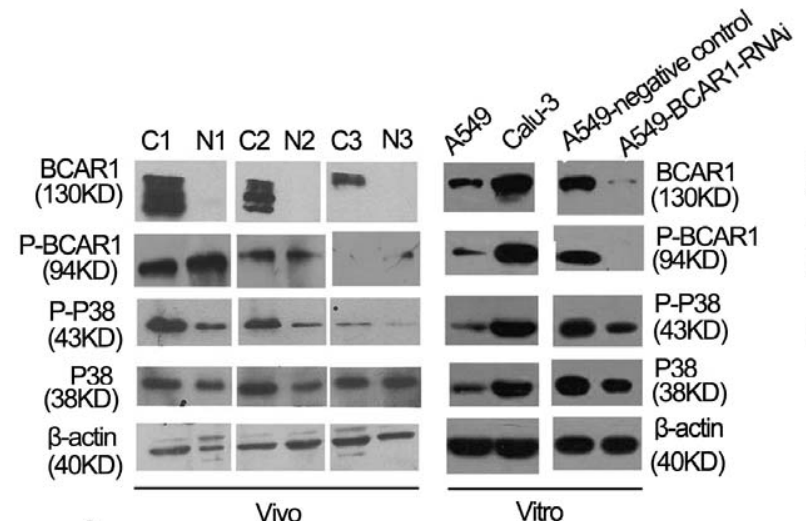

A

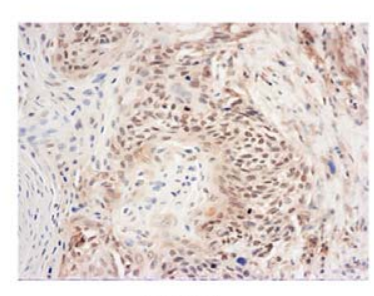

BCAR1



P38

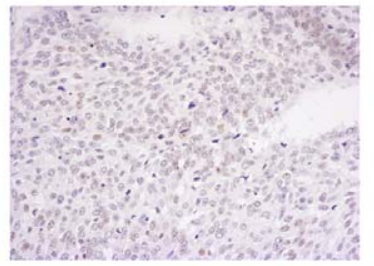

P-BCAR1

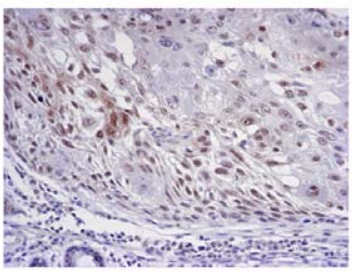

P-P38

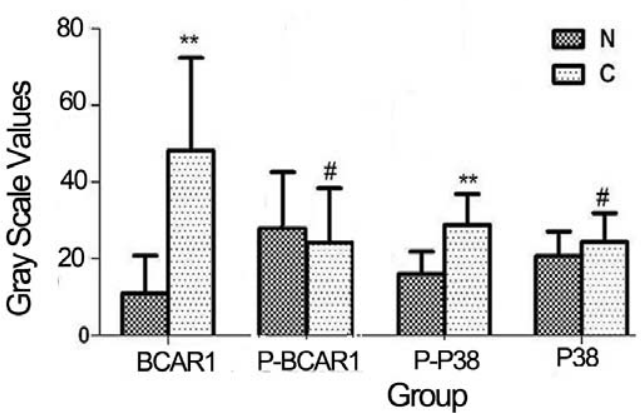

B

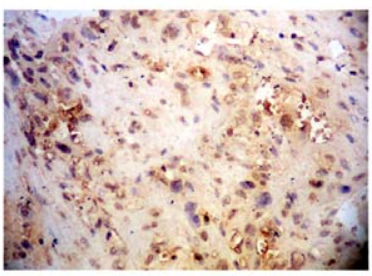

TUNEL Assay

Figure 1. BCAR1, phospho-BCAR1, p38 and phospho-p38 expression in NSCLC tissues and cells. A: Immunoblotting indicated either BCAR1 or phospho-p38(Thr180/Tyr182) levels in three NSCLC tissues (C) were significantly higher than in the adjacent normal tissues (N). But phospho-BCAR1 (Tyr410) and p38 levels in NSCLC and the adjacent normal tissues were similar. BCAR1, phospho-BCAR1, p38 and phospho-p38 expressions were also detected in A549 and Calu-3 NSCLC cell line by using Immunoblotting assay. BCAR1 knockdown causes the appreciable reduction of phospho-BCAR1 and phospho-p38 levels in A549 cells. B: Gray scales analysis of immunoblotting also suggested BCAR1 and phospho$\mathrm{p} 38$ (Thr180/Tyr182) levels were significantly higher in NSCLC than in the normal adjacent tissue $(48.18 \pm 24.7$ vs $10.97 \pm 9.8, P<0.001 ; 16.03 \pm 5.8$ vs $28.82 \pm 8.0, P<0.001)$. However, phospho-BCAR1 (Tyr410) and $\mathrm{p} 38$ had not the trend $(20.72 \pm 6.4$ vs $24.37 \pm 7.5, P=0.22 ; 25.3 \pm 11.2$ vs $27.8 \pm 15.2$, $P=0.476)$. C: IHC suggested the expressions of BCAR1 (either in the nucleus, the cytoplasm), phospho- BCAR1 (prone to locate in the cytoplasm), phospho-p38 (prone to locate in the nucleus), p38 (prone to locate in the cytoplasm) and apoptotic bodies. Note: $\mathrm{N}$ (adjacent normal tissue); $\mathrm{C}$ (NSCLC tissue); ** $(P<0.001)$; \# $(P>0.05)$.

doi:10.1371/journal.pone.0036124.g001

analysis revealed phospho-p38 levels were significantly higher in NSGLC than in the normal adjacent tissues (16.03 \pm 5.8 vs 28.82 $\pm 8.0, P<0.001$; Figure 1b). However, p38 had not the trend (20.72 \pm 6.4 vs $24.37 \pm 7.5, P=0.22$ ) (Figure 1b). Besides, IHC suggested positive rate of either BCAR1 or phospho-p38 was substantially higher in NSGLC than in normal tissue $(P<0.001$ and $\mathrm{P}<0.001$, respectively) (table 1). However, p38 had not such trend $(P=0.62)$.

We found percentages of phospho-p38 positive cells was significantly and positively correlated with those of BCAR 1 positive cells, in all the 182 NSCLC tissues (Spearman's rho, correlation coefficient $=0.811, \mathrm{p}<0.001$; Figure $2 \mathrm{~b})$. And percentages of phospho-p38 positive cells in BCAR1 high-expressed tissues were significantly higher than in BCAR1 low-expressed tissues (Mann-whitney $\mathrm{U}$ test $\mathrm{z}=-3.689 P<0.001$; Figure $2 \mathrm{~b}$ ). However, there was not any correlation between phospho-BCAR 1 and phospho-p38 levels $(\mathrm{P}=0.892)$.
In an attempt to depict the strong correlation between BCAR1 positive and phospho-p38 positive cells, we presented the sequential sections stained in one case for BCAR1, phosphoBCAR1, phospho-p38 and p38, which demonstrated that the cells over-expressing BCAR1 also have a higher level of phospho-p38 (Figure 2c).

BCAR 1 levels in Calu-3 cells were detectably higher compared with A549 cells (Figure 1a). Intriguingly, Figure 1a suggested phospho-p38 levels also had the same trend. Furthermore, BCARl knockdown also causes the appreciable reduction of phospho-p38 abundance in A549 cells (Figure 1a).

\section{Discussion}

BCAR1 as an adapter protein localizes to chromosome 16q22$\mathrm{q} 23^{7}$, and mainly consists of four functional portions including an amino-terminal Src homology 3 (SH3) domain, a Src-binding 


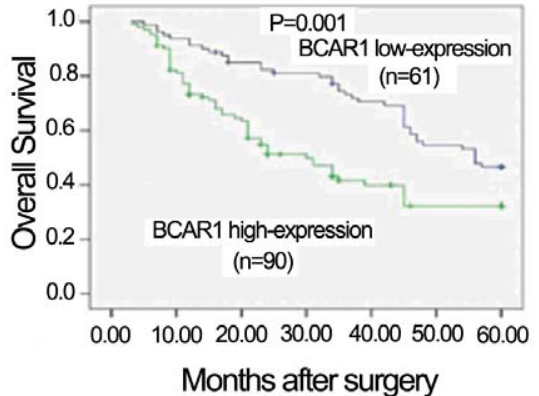

A

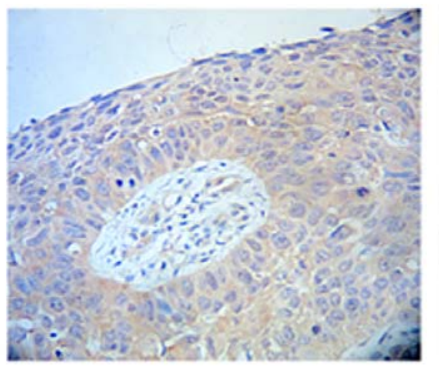

BCAR1

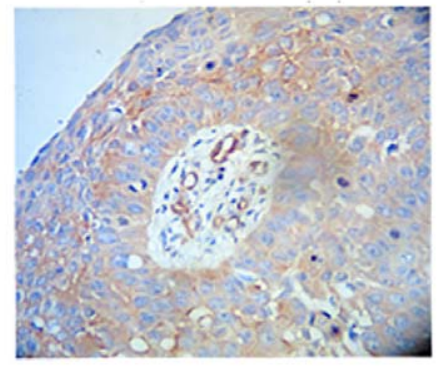

P-P38

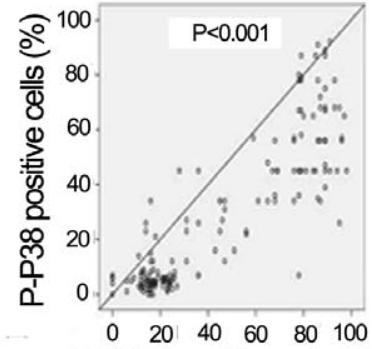

BCAR1 positive cells (\%)

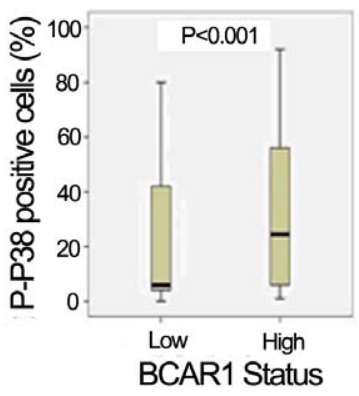

B

BCAR1 Status

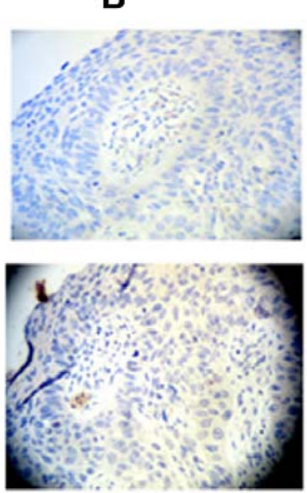

P38

\section{P-BCAR1}

C

Figure 2. High levels of BCAR1 predict poorer prognosis, and are correlated to activation of p38. A: The survival rate of BCAR1 highexpressed group was significantly lower than of low-expressed group $(P=0.001)$. B: Phospho-p38 positive cells was significantly and positively correlated with percentages of BCAR1 positive cells, in the 182 NSCLC tissues (Spearman's rho, correlation coefficient $=0.811, p<0.001$ ). And percentages of phospho-p38 positive cells in BCAR1 high-expressed tissues were significantly higher than in low-expressed tissues (Mann-whitney $U$ test $z=-3.689 P<0.001)$. C: The sequential sections were stained for BCAR1, phospho-BCAR1, phospho-p38 and p38, which demonstrated that the cells over-expressing BCAR1 also have a higher level of phospho-p38 $(\times 200)$. Either phospho-BCAR1 or p38 was slightly positive.

doi:10.1371/journal.pone.0036124.g002

domain (SBD), a large substrate domain (SD) and a helix-loophelix domain (HLH) $[12,13]$. BCAR1 locates ubiquitously in vitro and vivo $[14,15,16]$, and is involved in various cellular processes including migration, chemotaxis, apoptosis, cell cycle, differentiation and so forth $[17,18]$.

Thus far, very few studies focused on the carcinogenesis of BCAR1 in lung cancer. Wei et al. [19] suggested that anchorageindependent phosphorylation of BCAR 1 protected lung adenocarcinoma cells from anoikis. Recently, our study suggested that serum BCARl levels were significantly higher in NSCLC than in the control group, increased gradually with the progression of tumor staging, and decreased after removal of the malignant lesions [9]. As a result, we presumed a novel oncogenic role of BCAR1 in NSCLC. Herein, we aimed to verify the clinical implications of BCAR1 overexpression and NSCLC, and to elucidate the carcinogenetic mechanisms of BCAR1 in NSCLC. As expected, we found BCAR1 protein is especially abundant in NSCLC cells and tissues. And our studies in vivo and vitro showed the close correlation between BCAR 1 expression and activation of p38 MAPK. Although prior studies have shown that tyrosine phosphorylation of BCARl can be critical for downstream signaling [20], our study indicated that phospho-BCARl(Tyr410) was detected in only 34 of the 182 cases, and not correlated with clinical-pathological characteristics. Thus far, numerous sites of human BCAR1 phosphorylation are found as: tyrosine residues aa 12, 128, 165, 192, 222, 224, 234, 249, 267, 287, 306, 327, 362, $372,387,410,653,664,666$; serine residues aa 134, 139, 292, 437, 639; and threonine residues aa 269, 326, 385. We presume that some specific sites of phosphorylation are critical for the signal cascades of BCAR1 in NSCLC. Intriguingly, adjacent normal tissues show much higher levels of phospho-BCAR 1 than of BCAR1 proteins (Figure 1a). We also confirmed the inhibition of phospho-BCAR 1 along with BCAR 1 knockdown in an attempt to verify the efficacy of this antibody. We presume that a special enzyme in lung tissues specifically decomposes Non-phosphoBCAR1, however, phospho-BCAR 1 can survive. And all the abovementioned hypotheses deserve further more investigations.

Our experiments in vitro demonstrated that BCAR1 knockdown in A549 cells caused cell growth arrest, cell cycle arrest and cell migration inhibition. Although BCAR1 knockdown in A549

Table 2. Multivariate regression analysis in predicting the overall survival of NSCLC patients.

\begin{tabular}{llll}
\hline & & & \\
\hline Variable & Hazard ratio & $\begin{array}{l}\mathbf{9 5 \%} \text { Cofidence } \\
\text { interval }\end{array}$ & $P$-value \\
\hline BCAR1 expressed levels & 1.777 & $1.064-2.968$ & 0.028 \\
Phospho-BCAR1 levels & 1.001 & $0.413-2.423$ & 0.999 \\
Age & 0.960 & $0.643-1.433$ & 0.843 \\
Gender & 0.778 & $0.493-1.225$ & 0.278 \\
Nodal status & 1.277 & $1.018-1.668$ & 0.040 \\
Tumor size & 1.113 & $0.748-1.655$ & 0.597 \\
Differentiation & 1.449 & $0.753-2.794$ & 0.267 \\
TNM stage & 1.298 & $1.068-1.588$ & 0.007 \\
Histology & 1.103 & $0.689-1.543$ & 0.882 \\
\hline doi:10.1371/journalpone.0036124.t002 & &
\end{tabular}

doi:10.1371/journal.pone.0036124.t002 


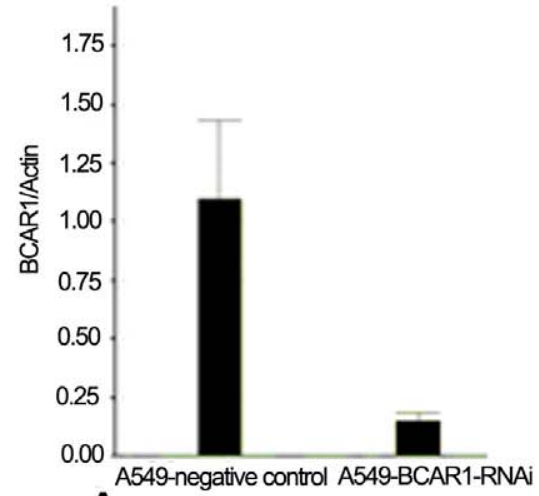

A

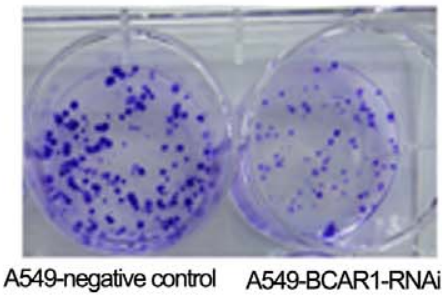

B

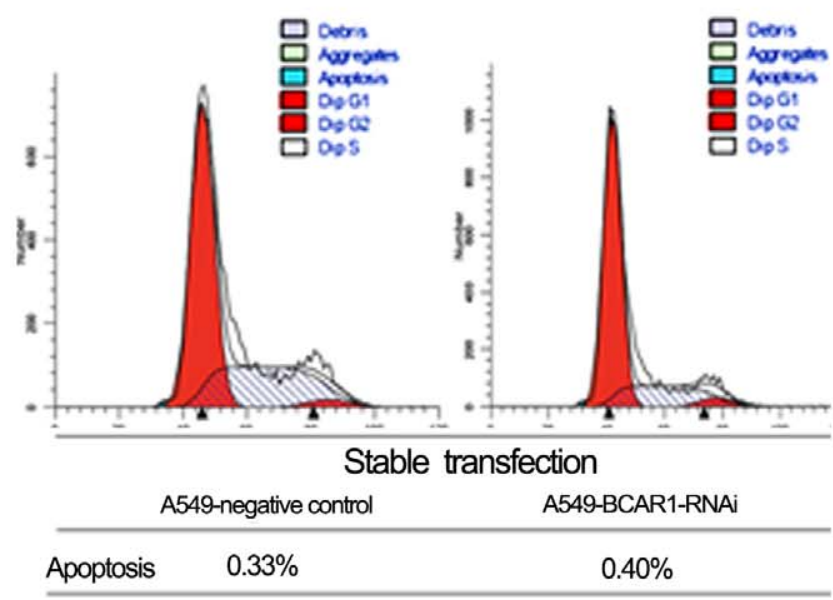
(Cells/visual field)

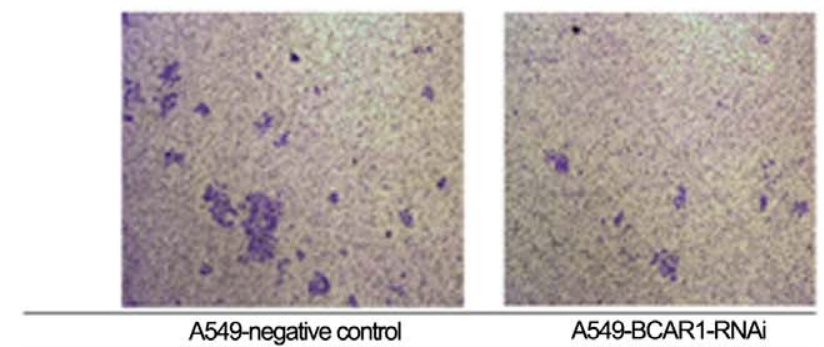

Cell Counting

129

53

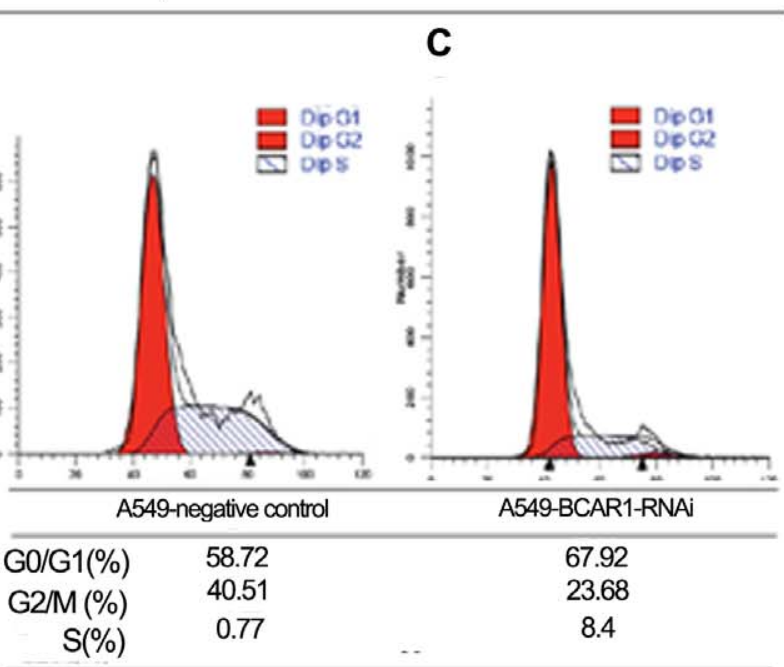

D

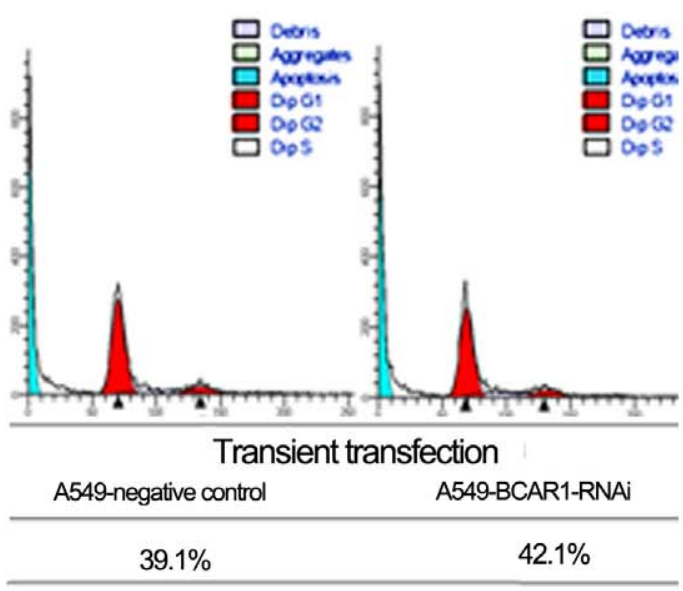

E

Figure 3. In A549 cells, BCAR1 knockdown caused cell growth arrest, cell migration inhibition, cell cycle arrest, but not apoptosis. A: At least an 80\% reduction in mRNA of BCAR1 in A549-BCAR1-RNAi cells was confirmed by real-time RT-PCR. B: Colony forming units of A549-BCAR1RNAi were detectably less than of A549-negative control cells, eleven days after plating. C: Cell migration assay suggested BCAR1 knockout prevented cell migration of A549-BCAR1-RNAi cells. D: Flow cytometry indicated BCAR1 knockout also caused cell cycle arrest of A549-BCAR1-RNAi cells. E: Flow cytometry indicated BCAR1 knockout did not increase apoptosis rate following either transient (39.1\% vs $42.1 \%)$ or stable transfection $(0.33 \%$ vs $0.4 \%)$ in A549 cells.

doi:10.1371/journal.pone.0036124.g003

cells did not cause cell apoptosis, there was an appreciable and inverse correlation between BCARl and apoptotic index in NSCLC tissues. We do not know the reasons for discrepancy between NSCLC cell and tissue. Additionally, our study in vivo demonstrated that BCARl levels were significantly and inversely correlated with tumor differentiation, by either counting positive percents of cells (Kruskal-Wallis test, $P=0.010$ ) or evaluating stained intensity (achromasy $=0, \quad$ stramineous $=1, \quad$ buffy $=2$, brown $=3$; Kruskal-Wallis test, $P=0.014$ ). Kaplan-Meier curve also indicated that higher levels of BCARl predicted poorer 
prognosis in 151 cases with valid follow up data. All the aforementioned experiments suggested BCAR1 had a crucial role in carcinogenesis. Besides, BCAR1 is known as Src substrate, and Src inhibitor AZD0530 can also result in significant inhibition of cell migration and matrigel invasion in lung cancer cells [21], which potentially supports the carcinogenesis of Src/BCARl axis.

Using immunoblotting, Greenberg et al. [22] found that only activated p38 MAPK was consistently increased in NSCLG in comparison with normal tissue, suggesting an additional role for this pathway in malignant cell growth or transformation. Indeed, numerous studies had unveiled the carcinogenetic activities of $\mathrm{p} 38$, including stimulation of proliferation and migration [1,23,24]. Matsuda $\mathrm{K}$ et al. [23] found that EGF promotes proliferation via p38 MAPK signaling cascades in ASPG-1, PANG-1 and T3M4 pancreatic cancer cell lines, and p38 MAPK inhibitor SB203580 can inhibit EGF-stimulated mitogenesis. Besides, the p38 MAPK pathway has been implicated to play an important role in endothelial cell migration because inhibiting p38MAPK activity down-regulates VEGF-stimulated migration [24]. Recently, Wendt et al.'s study [25] demonstrated that increasing expression of either the full-length or just the carboxyl terminus of BCAR 1 in mammary epithelial cells increased p38 activation. Intriguingly, in 182 NSGLC tissues, we found there was a close correlation between the expression of BCAR1 and phospho-p38. By evaluating stained intensity of phospho-p38 and BCAR1 (achromasy $=0$, stramineous $=1$, buffy $=2$, brown $=3$, " $<2$ scores" were classified as low expression, otherwise as high expression), we found the abundance of phospho-p38 was also significantly and positively correlated with that of BCAR1 (Spearman's rho, $\mathrm{p}<0.001)$. Besides, BCAR1 knockdown also caused the appreciable reduction of phospho-p38 levels in A549 cells. However, the

\section{References}

1. Rooney C, Sethi T (2011) The epithelial cell and lung cancer: the link between chronic obstructive pulmonary disease and lung cancer. Respiration 81: 89-104.

2. Ulahannan SV, Brahmer JR (2011) Antiangiogenic agents in combination with chemotherapy in patients with advanced non-small cell lung cancer. Cancer Invest 29: 325-337.

3. Huang C, Liu D, Masuya D, Nakashima T, Kameyama K, et al. (2005) Clinical application of biological markers for treatments of resectable non-small-cell lung cancers. Br J Cancer 92: 1231-1239.

4. Reynolds AB, Roesel DJ, Kanner SB, Parsons JT (1989) Transformation-specific tyrosine phosphorylation of a novel cellular protein in chicken cells expressing oncogenic variants of the avian cellular src gene. Mol Cell Biol 9: 629-638.

5. Kanner SB, Reynolds AB, Parsons JT (1991) Tyrosine phosphorylation of a 120kilodalton pp60src substrate upon epidermal growth factor and platelet-derived growth factor receptor stimulation and in polyomavirus middle-T-antigentransformed cells. Mol Cell Biol 11: 713-720.

6. Tikhmyanova N, Little JL, Golemis EA (2010) CAS proteins in normal and pathological cell growth control. Cell Mol Life Sci 67: 1025-1048.

7. Brinkman A, der Flier Sv, Kok EM, Dorssers LC (2000) BCAR1, a human homologue of the adapter protein p130Cas, and antiestrogen resistance in breast cancer cells. J Natl Cancer Inst 92: 112-120.

8. Dorssers LC, Grebenchtchikov N, Brinkman A, Look MP, van BSP, et al. (2004) The prognostic value of BCARl in patients with primary breast cancer. Clin Cancer Res 10: 6194-6202.

9. Deng B, Huang W, Tan QY, Fan XQ Jiang YG, et al. (2011) Breast cancer anti-estrogen resistance protein 1 (BCAR $1 / \mathrm{p} 130 \mathrm{cas}$ ) in pulmonary disease tissue and serum. Mol Diagn Ther 15: 31-40.

10. Cuadrado A, Nebreda AR (2010) Mechanisms and functions of p38 MAPK signalling. Biochem J 429: 403-417.

11. Ji C, Ren F, Xu M (2010) Caspase-8 and p38MAPK in DATS-induced apoptosis of human CNE2 cells. Braz J Med Biol Res 43: 821-827.

12. Kim W, Seok KY, Soo KJ, Shin NY, Hanks SK, et al. (2008) The integrincoupled signaling adaptor p130Cas suppresses Smad3 function in transforming growth factor-beta signaling. Mol Biol Cell 19: 2135-2146.

13. Chodniewicz D, Klemke RL (2004) Regulation of integrin-mediated cellular responses through assembly of a CAS/Crk scaffold. Biochim Biophys Acta 1692: 63-76. underlying mechanisms deserve further investigations. Additionally, Figure la demonstrated BCARl knockdown slightly decreased expression of total p38 in A549 cells. Furthermore, adjacent normal tissues had low BCAR1, but not a decreased level of total p38. We think that the expression of total p38 is regulated by the other signal cascades except for BCARl in NSCLC.

Collectively, BCAR1 is associated with poor prognosis of NSCLC patients. BCAR1 knockdown experiments in A549 cells strongly supported the carcinogenesis of BCARl in NSCLC, probably via the activation of $\mathrm{p} 38 \mathrm{MAPK}$. And we presume BCAR1 may be a potential therapeutic target for NSCLC. However, the further investigations are required immediately as: (i)Mechanistic link between BCAR1 and phospho-p38 should be studied; (ii) Stronger evidence should be provided to verify carcinogenesis of BCAR1 through activation of p38; (iii) The other key signaling molecules altered or activated by BCAR1, other than p38, should be evaluated.

\section{Acknowledgments}

We appreciate Prof. Li and his staff from the pathological department of Daping Hospital, who helped us conduct IHC and review slides. We also appreciate the anonymous reviewers and Dr. Keiran Smalley for their excellent comments and editorial review, respectively.

\section{Author Contributions}

Conceived and designed the experiments: BD. Performed the experiments: WH BD RW QT YH YJ JZ. Analyzed the data: WH BD RW. Contributed reagents/materials/analysis tools: WH BD RW QT YH YJ JZ. Wrote the paper: WH BD.

14. Sakai R, Iwamatsu A, Hirano N, Ogawa S, Tanaka T, et al. (1994) A novel signaling molecule, p130, forms stable complexes in vivo with v-Crk and v-Src in a tyrosine phosphorylation-dependent manner. EMBO J 13: 3748-3756.

15. Honda H, Oda H, Nakamoto T, Honda Z, Sakai R, et al. (1998) Cardiovascular anomaly, impaired actin bundling and resistance to Src-induced transformation in mice lacking p130Cas. Nat Genet 19: 361-365.

16. Defilippi P, Di SP, Cabodi S (2006) p130Cas: a versatile scaffold in signaling networks. Trends Cell Biol 16: 257-263.

17. Takino T, Tamura M, Miyamori H, Araki M, Matsumoto K, et al. (2003) Tyrosine phosphorylation of the CrkII adaptor protein modulates cell migration. J Cell Sci 116: 3145-3155.

18. Kim W, Kook S, Kim DJ, Teodorof C, Song WK (2004) The 31-kDa caspasegenerated cleavage product of $\mathrm{p} 130 \mathrm{cas}$ functions as a transcriptional repressor of E2A in apoptotic cells. J Biol Chem 279: 8333-8342.

19. Wei L, Yang Y, Zhang X, Yu Q (2002) Anchorage-independent phosphorylation of p130(Cas) protects lung adenocarcinoma cells from anoikis. J Cell Biochem 87: 439-449.

20. Brabek J, Constancio SS, Siesser PF, Shin NY, Pozzi A, et al. (2005) Crkassociated substrate tyrosine phosphorylation sites are critical for invasionand metastasis of SRC-transformed cells. Mol Cancer Res 3: 307-315.

21. Purnell PR, Mack PC, Tepper CG, Evans CP, Green TP, et al. (2009) The Src inhibitor AZD0530 blocks invasion and may act as a radiosensitizer inlung cancer cells. J Thorac Oncol 4: 448-454.

22. Greenberg AK, Basu S, Hu J, Yie TA, Tchou-Wong KM, et al. (2002) Selective p38 activation in human non-small cell lung cancer. Am J Respir Cell Mol Biol 26: $558-564$.

23. Matsuda K, Idezawa T, You XJ, Kothari NH, Fan H, et al. (2002) Multiple mitogenic pathways in pancreatic cancer cells are blocked by a truncated epidermal growth factor receptor. Cancer Res 62: 5611-5617.

24. Yu J, Bian D, Mahanivong C, Cheng RK, Zhou W, et al. (2004) p38 Mitogenactivated protein kinase regulation of endothelial cell migration depends on urokinase plasminogen activator expression. J Biol Chem 279: 50446-50454.

25. Wendt MK, Smith JA, Schiemann WP (2009) pl30Cas is required for mammary tumor growth and transforming growth factor-beta-mediated metastasis through regulation of Smad2/3 activity. J Biol Chem 284: $34145-34156$ 